Itinéraires Itinéraires

Littérature, textes, cultures

2019-2 et $3 \mid 2019$

Corps masculins et nation : textes, images, représentations

\title{
Los placeres ocultos (1977) d'Eloy de la Iglesia : un manifeste contre l'hétérocentrisme au tout début de la transition démocratique espagnole
}

Los placeres ocultos (1977) by Eloy de la Iglesia: A Manifesto against

Heterocentrism in the Early Days of the Spanish Democratic Transition

\section{Emmanuel Le Vagueresse}

\section{OpenEdition}

\section{Journals}

Édition électronique

URL : http://journals.openedition.org/itineraires/7001

DOI : $10.4000 /$ itineraires. 7001

ISSN : 2427-920X

Éditeur

Pléiade

Référence électronique

Emmanuel Le Vagueresse, «Los placeres ocultos (1977) d'Eloy de la Iglesia : un manifeste contre

I'hétérocentrisme au tout début de la transition démocratique espagnole », Itinéraires [En ligne], 2019-2 et 3 | 2019, mis en ligne le 13 décembre 2019, consulté le 15 décembre 2019. URL : http://

journals.openedition.org/itineraires/7001 ; DOI : 10.4000/itineraires.7001

Ce document a été généré automatiquement le 15 décembre 2019.

\section{$(1) \Theta \Theta$}

Itinéraires est mis à disposition selon les termes de la licence Creative Commons Attribution - Pas d'Utilisation Commerciale - Pas de Modification 4.0 International. 


\section{Los placeres ocultos (1977) d'Eloy de la Iglesia : un manifeste contre l'hétérocentrisme au tout début de la transition démocratique espagnole}

Los placeres ocultos (1977) by Eloy de la Iglesia: A Manifesto against

Heterocentrism in the Early Days of the Spanish Democratic Transition

Emmanuel Le Vagueresse

« Croyez-moi, la patrie est mère de tous les

vices. "

Luis Buñuel« Si quand les nègres sont persécutés, tu ne te sens pas nègre, si quand les femmes sont méprisées, ou les ouvriers, tu ne te sens pas femme ou ouvrier, alors, toute ta vie, tu auras été

un pédé pour rien." Jean Genet

Le cinéaste espagnol Eloy de la Iglesia (1944-2006) a toujours été engagé contre la doxa et les dogmes, quels qu'ils soient, moraux, sensuels ou sexuels, mais aussi politiques, nationaux ou patriotiques. Avec ce long métrage de fiction, son dixième à l'époque, Los placeres ocultos - « Les Plaisirs cachés », littéralement, en hommage au Cernuda poète de Los placeres prohibidos (1931) [ "Les Plaisirs interdits "] ${ }^{1}-$, le réalisateur brave plus ouvertement que jamais la censure ${ }^{2}$ (post-)franquiste moribonde, mais qui bouge encore - elle ne sera définitivement abolie que le 11 novembre 1977 -, en proposant une histoire de bourgeois madrilène homosexuel dans le placard, qui refuse de faire partie du mouvement gay naissant, Eduardo (Simón Andreu), entouré de prostitués, issus du lumpenprolétariat ou peu s'en faut ${ }^{3}$. Ce banquier tente soudain de prendre sous son aile un jeune des cités, Miguel (Tony Fuentes), pour le faire progresser dans la 
société, en sublimant - même si c'est parfois à grand-peine - son désir pour ce garçon hétérosexuel, afin de le faire accéder aux sphères supérieures de la réussite sociale.

2 Film d'une heure et trente-quatre minutes en couleurs, interdit à l'époque aux moins de 18 ans, écrit par Eloy de la Iglesia, Rafael Sánchez Campoy et Gonzalo Goicoechea, et retenu plusieurs semaines par la censure, il fut présenté sur les écrans espagnols en même temps que le Viridiana de Luis Buñuel de 1961 - tout un symbole de fin d'une époque répressive-, soit le 14 avril 1977 à Madrid, au cinéma Postas (date d'autorisation: 15 mars 1977). La sortie de Los placeres ocultos - qui totalisa 170000 spectateurs $^{4}$ - est une date clef dans la représentation cinématographique commerciale de la " question gay ", à un moment où tout semble devenir possible.

Questionnant l'hétérocentrisme de la nation espagnole au tout début de la transition démocratique via un cinéma faussement mainstream et avec force «stars» au casting (ici Simón Andreu, Charo López, et plus furtivement le travesti Paco España), ce filmmanifeste est reçu non sans quelques réticences par la presse, même si la tonalité des journaux progressistes est plus positive, la sortie de ce long métrage se doublant aussi d'un relatif succès d'audience. Il annonce en fait la profusion de films plus ou moins militants, mais aussi voyeurs, sur le sujet de l'homosexualité - essentiellement masculine - jusqu'à la fin de ladite Transition. Et ce film fait de l'acceptation de l'homosexuel «moyen » - et plus seulement de la «folle » des cabarets, qui n'inquiète finalement guère la nation, car cette folle est immédiatement repérable et cantonnée majoritairement au ghetto du monde du spectacle - le symptôme de la nouvelle liberté de tout un pays en voie vers la démocratie ${ }^{5}$.

4 Nous étudierons d'abord, dans cet article, comment Eloy de la Iglesia propose dans son film une vision de l'homosexualité "intégratrice», qui acquiert une résonance immédiatement politique, dans une époque de changements tous azimuts, mais où l'homosexualité était encore officiellement réprimée ${ }^{6}$. Nous insisterons ensuite sur la manière dont, justement, le réalisateur joue sur la masculinité des différents protagonistes de cette histoire, entre un hétérosexuel sans ambiguïté et un homosexuel viril, comme si cette masculinité partagée était obligatoire pour « intégrer » les gays à la nation, en cette période du tout début de la transition démocratique, après des décennies de moqueries filmiques, entre autres, sur des homosexuels féminisés ${ }^{7}$. Enfin, si nous décidons d'aller un peu plus loin que de passer simplement le rasoir d'Ockham, nous annonçons que nous nous pencherons sur l'ambiguïté, ou en tout cas l'étrange spécificité de ce film, comme bientôt d'autres du même réalisateur, quant au voyeurisme de certaines images.

\section{Vers une homosexualité « intégratrice »?}

5 Certains photogrammes présentent en effet une sorte de « racolage» du spectateur, tant gay que straight, car sont exhibés dans Los placeres ocultos bien des corps dénudés, féminins et masculins, ce qui est à la fois une limite du cinéma politique d'Eloy de la Iglesia - qui est, donc, aussi, commercial-, mais en même temps une stratégie d'entrisme, par le biais de cette complaisance envers les instincts spectatoriels, en ces débuts du cinéma dit de destape - ou «déshabillage »- qui cache et permet simultanément de faire passer plus aisément, sans doute, un « message » militant ${ }^{8}$. 
6 Si l'on visionne le film, en effet, on se rend compte que dès le pré-générique est montré un corps de jeune homme nu, des images qui succèdent très vraisemblablement à une scène d'amour non montrée, entre un homme présenté comme bourgeois et fortuné, sans doute dandy, vu son intérieur richement décoré, et ce prostitué, puisque le garçon se fait payer à la fin de la séquence. On n'avait encore jamais vu cela dans le cinéma espagnol, toujours officiellement sous la coupe de la censure, nécessitant donc, plutôt, des œuvres ad usum Delphini, c'est-à-dire (auto)censurées ou expurgées, au moins en apparence.

7 Et à propos de censure et d'ellipse nécessaires, ce pré-générique, qui ouvre sur l'instant suivant une scène de lit homosexuelle traitée par une espèce d'ellipse absolue, ressortit à ce que Linda Williams appelle des "points de suspension » qui ne font pourtant, si l'on y réfléchit bien, que souligner davantage cette scène in absentia, comme souvent les points de suspension dans tout " discours" ${ }^{\text {». }}$

8 Au-delà de ce pré-générique audacieux et même révolutionnaire - même si « l'art agit mal et agit peu ", selon le lucide Maurice Blanchot dans son Espace littéraire ([1955] $1988: 282$ ) -, surgit tout de suite une opposition entre la grande bourgeoisie de celui qui va s'avérer être le directeur d'une banque de Madrid à l'époque de la diégèse, le prénommé Eduardo, et la cité de banlieue où vit le personnage du jeune Miguel - ce n'est pas le prostitué vu avant les crédits initiaux et, pour l'instant, Miguel et Eduardo ne se sont pas encore rencontrés. Et ce, même si ce garçon n'appartient pas, stricto sensu, au lumpenprolétariat, puisqu'il suit des cours dans une école de type "secrétariat", mais s'inscrit a priori dans le fantasme du jeune de banlieue mal dégrossi, brut(e) et sexy ${ }^{10}$. Cette opposition sociale est flagrante, car la mère du jeune homme, dont l'époux les a quittés brusquement bien longtemps auparavant, est une femme de ménage qui vit dans un intérieur modeste contrastant avec celui d'Eduardo. Et ses trois fils mâles cohabitent, voire s'entassent, dans une même chambre ${ }^{11}$.

On apprend aussi que cette famille vient de la région de Jaén, province pauvre du sud de l'Espagne, ce qui est peut-être un cliché « didactique » de la part du réalisateur, mais pas plus que le gay bourgeois et potentiellement corrupteur - ce terme et ses polyptotes reviennent souvent dans le film -, de jeunes gens, avec son argent, au début du film : potentat en habit de prix, comme dans toutes les comédies espagnoles d'avant la fin du franquisme, sauf que dans ce film-ci, justement, tout va changer.

10 Rapidement, le doute n'est plus permis aux yeux du spectateur quant à l'hétérosexualité du jeune protagoniste, qui est doté d'une fiancée, Carmen (Beatriz Rossat), au prénom connoté «espagnol» un peu clichéique, là encore. Miguel ne semble pas entretenir de relations sexuelles masculines, à la différence de certains de ses amis ou connaissances du quartier, dont un certain Nes (Ángel Pardo) que l'on voit, de manière quasi documentaire, en un ballet de gigolos pour hommes relativement aisés, sur le Paseo de Recoletos, à la sortie de la station homonyme de trains de banlieue $^{12}$. C'est devant un magasin de motos qu'Eduardo aperçoit Miguel, le croyant peut-être accessible - au moins par l'argent - et fomentant un stratagème pour l'attirer à lui, à savoir lui proposer un travail en même temps qu'une formation, au nom d'une mystérieuse organisation philanthropique qui détecterait les jeunes pousses prometteuses. On notera tout de suite que ce qui semble relier les individus entre eux est ici l'argent, que ce soit celui donné par le client, comme au début du film, ou celui reçu par le prostitué, ce qui sous-entend un rapport entre les classes faussé par cet 
argent, qui peut acheter, sinon les sentiments, du moins les corps : vision d'un créateur férocement de gauche comme l'était le réalisateur.

11 On pense alors aux railleries de l'écrivain italien Curzio Malaparte sur les rapports sexuels nécessairement tarifés, dans le milieu homosexuel, entre bourgeois soi-disant de gauche et prolétaires, le bourgeois progressiste étant même, ici, davantage Eloy de la Iglesia en réflexion sur lui-même, sans doute, que son protagoniste Eduardo ${ }^{13}$ :

Ces nobles Narcisses qui, jusqu'alors, s'étaient posés en esthètes décadents [...] se posaient maintenant en esthètes marxistes [...], empruntant les motifs de leur nouvel esthétisme à Marx, à Lénine, à Staline, à Chostakowitch [sic], et parlaient avec mépris du conformisme sexuel « capitaliste » [...]. Ils se figuraient avoir trouvé dans le communisme un point de rencontre avec les éphèbes prolétariens, une complicité, un nouveau pacte, non seulement sexuel, mais de nature morale et sociale ${ }^{14}$.

Dans l'une des premières scènes-clefs du film, Raúl (Antonio Corencia), que l'on comprend être un ex-protégé et amant d'Eduardo, gay quant à lui, pousse ce dernier à faire son coming out, pour faire avancer la société, à un moment où les libertés peuvent réapparaitre, mais où il faut aussi les prendre à bras-le-corps, avec détermination et courage. Mais Eduardo ne paraît pas intéressé par ce combat collectif, préférant la satisfaction de ses désirs personnels, sans jamais vouloir les hausser au niveau d'une lutte en commun ${ }^{15}$.

En tout cas, Miguel, lui, semble prendre Eduardo pour un philanthrope, puisque ce dernier lui donne du travail, sur fond de contrastes, là encore, entre la cité de banlieue où il le reconduira un peu plus tard dans le film ${ }^{16}$ et l'appartement bourgeois où il l'invite. Lorsque Miguel lui apprend qu'il a une fiancée, Eduardo est apparemment surpris, puis déçu, mais il se reprend immédiatement et maintient sa collaboration de travail - à savoir, que Miguel lui tape des textes à la machine -, même si le spectateur a l'impression que cette relation pourrait même aller plus loin et qu'Eduardo a toujours en tête que Miguel est « achetable » et, même doté d'une petite amie, qu'il peut se muer en un gay for pay ${ }^{17}$.

Lorsque Miguel accepte de se faire inviter, sans son amie, au spectacle par Eduardo cette relation d'achat/vente entre les deux hommes saute aux yeux du spectateur, le bourgeois abusant de sa situation avec ce jeune sans le sou. Il n'est pas inutile d'observer que l'invitation se fait dans un cabaret où les deux hommes assistent à un numéro de travesti, ce dont nous reparlerons. Après le spectacle, le «maître » et l'«élève ", en quelque sorte, rencontrent deux prostituées qu'ils emmènent chez Eduardo, ce dernier montrant une fois de plus qu'il ne peut concevoir l'amour - en tout cas le sexe - que tarifé. Le maître et l'élève deviennent alors une sorte d'incarnation contemporaine de l'éraste et l'éromène ${ }^{18}$, l'éphèbe étant désiré ici par le vieil amoureux, le doute n'étant pas de mise, vu le regard posé par Eduardo sur Miguel, quant à la nature amoureuse, et non plus seulement sexuelle, de cette attirance.

Par la suite, le spectateur espagnol rentre, pour l'une des premières fois, dans un bar gay, constatant une nouvelle fois l'existence de rapports tarifés entre bourgeois et jeunes chaperos - ou " gigolos ", " taxi-boys » - comme un peu plus tard il rentrera dans des urinoirs ou un cinéma, typiques lieux de drague gay urbaine de l'époque, ce qui apporte un " effet de réel » aussi documenté qu'inédit et marquant à ces scènes. Il faut aussi noter que, au mitan du film, Eduardo, sans doute un peu gêné par ses propres manigances, révèle enfin à Miguel, à la fois sa stratégie et son attirance pour lui. Contre toute attente, le jeune homme lui rétorque qu'il avait déjà compris lesdites stratégie et 
attirance, venant du «quartier » - donc pas si naïf qu'Eduardo ne le croyait, faut-il comprendre -, et ajoute qu'il aurait été plus simple pour le banquier de se déclarer franchement. En effet, Miguel lui aurait dit non... : «y basta " (« et ça aurait suffi»), conclut-il, tout en s'éloignant brusquement et lui lançant un "¡Quita, mariquita!» («Bas les pattes, pédale!»), lorsque le banquier veut prendre son visage dans ses mains.

Quelques minutes après, le personnage de Rosa (Charo López), une femme d'environ trente-cinq ans qui couche régulièrement avec Miguel, comme on le voit au début du film, fait retour à l'écran, les rumeurs commençant à enfler quant à la prostitution homosexuelle de ce dernier. Jalouse, elle lui propose de l'entretenir, même si elle précise qu'elle n'a pas autant d'argent qu'Eduardo, ce qui replace les rapports, sexuels ou plus généraux, sous le sceau de l'argent. Mais la discussion la plus signifiante, à ce moment-là de l'économie du film, est celle entre Raúl et Miguel, une discussion où le bourgeois "révolutionnaire» qu'est Raúl lui parle de lutte pour l'égalité entre homosexuels et hétérosexuels, à la grande perplexité et même à la fureur, quelques secondes durant, du jeune homme, qui cesse néanmoins à la fin de la discussion d'être en colère ${ }^{19}$.

Mais le « réel » revient à la charge, face au volontarisme et à l'ouverture possibles de ce jeune hétérosexuel à la question gay - Miguel n'a pas l'air, en effet, aussi furieux que dans la scène du coming out d'Eduardo, malgré sa perplexité -, un réel qui s'invite via le guet-apens ourdi par Nes avec sa bande pour extorquer de l'argent à Eduardo en échange de leur silence, mettant au passage sens dessus dessous son bel appartement. On notera aussi la menace faite par Nes de sodomiser Eduardo, comme si, ainsi, sa propre masculinité était vaincue, faisant de lui un passif, voire une femme, alors que la masculinité du violeur, qui reste actif, donc viril, n'est absolument pas remise en cause par cette proposition.

\section{Des jeux sur les masculinités}

18 Miguel semble donc progresser dans sa vision de l'homosexualité de par sa relation, certes brisée, avec Eduardo, quoi qu'il ait pu lui lancer à la figure : en effet, on ressent son malaise quand il apprend cette agression et ces menaces envers son ex-protecteur, jusqu'à mentir à sa petite amie Carmen en prétextant un empêchement pour ne pas honorer son rendez-vous galant avec elle. C'est qu'il décide de venger Eduardo en frappant Nes, d'une part (ce qui ressortit à une action concrète en défense d'un gay, sinon de tous les gays espagnols), puis discute avec ses deux frères en prenant le parti des gays, là encore, face aux clichés de ses cadets sur l'homosexualité (ce qui ressortit à une prise de parole en défense d'un gay, sinon là encore de tous les gays espagnols).

La deuxième discussion entre Eduardo et Miguel - car ils se revoient, finalement, la rupture n'étant apparemment pas si grave - est fondamentale, car elle évoque le caractère "inné » de cette sexualité, et parce qu'elle montre une avancée encore plus nette dans l'acceptation de l'homosexualité de ce dernier, même si la route est encore longue pour Miguel, lequel lui propose néanmoins d'être son ami, du moment qu'il ne cherche pas à le convertir ${ }^{20}$, tout comme est longue, dans une autre direction, celle d'Eduardo avec Raúl, d'ailleurs, pour ce qui est de la visibilité et du combat collectifs en faveur de l'homosexualité. Une nouvelle opposition surgit alors, avec le chantage, quoiqu'elle s'en défende, de la part de Rosa envers Eduardo, laquelle Rosa le traite à 
l'envi de " gigolo » ou d'« inverti », ce qui, doublé du chantage de Nes et ses amis, est là pour rappeler aussi la pénalisation possible de l'homosexualité, même après la mort de Franco, et/ou les ravages probables du contrôle social.

Le rose n'est donc pas encore mis dans cette Espagne balbutiante de l'après-Franco, et c'est donc Nes, cette fois, qui se venge de Miguel avec sa bande, comme on le verra plus tard, en le frappant et en détruisant sa moto ${ }^{21}$, prolongement métonymique de sa virilité, tandis que, d'un autre côté, Rosa va détruire le jeune homme par la parole, ruinant sa réputation auprès des parents de Carmen en l'accusant d'être un prostitué, le père interdisant alors, désormais, à sa fille de revoir Miguel.

Dans l'avant-dernière scène du film, une ultime - pour le moment ? - discussion, qui tourne à la dispute, se fait alors entendre, cette fois dans la banque d'Eduardo, entre celui-ci et Miguel, qui arrive blessé de l'attaque de Nes et ses amis. Miguel l'interpellant d'un "¡Marica! » répété et lancé une seconde fois très fort ${ }^{22}$ dans le hall de la banque, donc dans l'espace professionnel même d'Eduardo, sinon un espace (quasi) public, la scène se déroule entre la gêne d'Eduardo face aux curieux ${ }^{23}$ et la nouvelle fuite de Miguel en pleurant, qui laisse la suite en suspens. Mais cette scène insiste néanmoins sur la sanction du contrôle social, sans doute plus que légal, de cet outing forcé pour le banquier ${ }^{24}$, voulu clairement par le jeune homme pour qu'il s'assume enfin, en une stratégie violente, encore discutée de nos jours par les différents collectifs gays quant à son efficacité, mais aussi quant à son éthique.

À la toute dernière minute du long métrage, on assiste à un retour à l'identique par rapport au début, puisque l'on voit Eduardo chez lui dans son habituel peignoir, alors que l'on sonne à la porte, et que celui-ci se déplace pour ouvrir. L'air heureux qu'il arbore en voyant, par le judas, qui a sonné, et son empressement, alors, à ouvrir la porte, laisse libre le spectateur d'interpréter comme il le souhaite ce finale, puisque l'image se fige sur le mot «FIN » au moment où la porte s'ouvre, laissant dans l'ombre l'identité du visiteur. On peut raisonnablement penser qu'il s'agit de Miguel et pas d'un nouveau prostitué parmi d'autres, vu la progression du personnage d'Eduardo au fil de la diégèse, mais aussi celle de Miguel, vers une meilleure intercompréhension de l'autre. L'avenir se veut alors plus optimiste sur les relations entre gays et straights, si l'on retient cette hypothèse cohérente de lecture du film ${ }^{25}$.

On a dit que ce film mettait en scène un homosexuel viril, à l'opposé de l'image de la «loca», la «folle» efféminée qui était alors quasiment la seule représentation acceptable du gay dans la cinématographie espagnole ${ }^{26}$. Ce choix de "normaliser» l'image de l'homosexuel de la part du réalisateur ${ }^{27}$ n'empêche pourtant, ni la présence d'une scène de cabaret avec chanteur travesti, le célèbre Paco España - de son vrai nom Francisco Moreira García -, dont le choix du pseudonyme pourrait aussi être l'objet d'une réflexion quant à la réappropriation subversive du nom même de la nation ${ }^{28}$, ni les limites, dans la diégèse, de cette masculinité obligée de l'homosexuel nouvelle manière.

En effet, si Eduardo emmène Miguel voir le show du transformiste (dans son propre rôle), c'est pour lui montrer que lui, Eduardo, n'est pas comme ce gay travesti en qui tout s'oppose à eux deux, mais qu'il est comme lui, Miguel, un homme, un vrai. Malgré cette stratégie spécieuse, lorsque les deux hommes reviennent au domicile d'Eduardo pour partager deux prostituées, Eduardo est incapable de satisfaire la « sienne » - sans grande surprise pour cette dernière, il faut le dire -, tandis que, dans la chambre d'à côté, Miguel fait l'amour à celle qui lui a été échue. 

préférences sexuelles, montre qu'Eduardo n'est pas dans la bonne optique pour se réaliser, et, après tout, il essaie de séduire un jeune homme qu'il sait alors être vraisemblablement hétérosexuel. C'est donc en sublimant, ensuite, ce désir et cet amour pour le jeune homme straight, notamment en l'aidant d'un seul point de vue professionnel, qu'Eduardo cessera de faire fausse route. Reste qu'il lui faudra encore progresser sur cette route de sa vérité pour trouver un homme qui, comme lui, aimera l'autre pour ce qu'il est, c'est-à-dire un homme homosexuel, et pas un jeune micheton hétéroflexible ${ }^{29}$.

\section{Un voyeurisme ambigu}

Enfin, le film joue tout de même, bien que restant militant, avec l'image profusément offerte de corps dénudés, tant masculins que féminins, d'ailleurs, ce qui ne va pas sans poser quelques problèmes éthiques, bien entendu, car ces corps sont présentés complaisamment dans le film ${ }^{30}$ et ce, même si, selon Foucault, le corps est la surface d'inscription des éléments. Pour ce qui est des corps féminins, on remarquera que, dès l'une des deux affiches du film - pas la principale, mais une seconde affiche, sans doute postérieure à la disparition de la censure -, un corps féminin, celui de la fiancée de Miguel, est visible sur la plage en maillot de bain, comme celui de Miguel, mais aussi celui de Nes torse nu (avec Eduardo, en peignoir, le touchant), tandis que l'affiche initiale montrait déjà Miguel torse nu et Rosa à peine plus vêtue au lit, avec en médaillon Eduardo, toujours en peignoir, mais bronzant simplement chez lui avec une lampe de bronzage ${ }^{31}$.

La stratégie est claire, dans les deux cas, et encore plus dans l'affiche post-censure ${ }^{32}$ : faire venir le plus de spectateurs possible, mâles hétérosexuels, mâles homosexuels, comme d'ailleurs femmes straight ou lesbiennes. En effet, le réalisateur voulait que ses films soient vus, rapportent, pour pouvoir en réaliser d'autres et faire passer ainsi le plus possible ses «messages" militants, et tromper une dernière fois les «crayons rouges » appointés de la dernière censure (post-)franquiste, encore bien homophobe.

Les exemples sont légion de ce voyeurisme, d'abord envers les jeunes femmes, comme Carmen, dont Miguel pétrit les seins, très tôt dans le film, ou Rosa, le corps de cette dernière étant copieusement exhibé. Mais, plus nouveau et subversif à l'époque, ce voyeurisme s'applique aussi aux garçons, depuis le pré-générique avec son plan, au bout de quelques secondes seulement, sur les fesses musclées du prostitué, puis plus tard sur le corps de Miguel après l'amour avec l'une des deux prostituées. Dans cette scène, nous voyons clairement le corps nu de Miguel avec les yeux d'Eduardo, ce qui constitue une transgression supplémentaire, le regard du spectateur moyen, à l'époque, surtout dans le cinéma commercial, n'étant pas celui d'un homme gay ${ }^{33}$.

Bien entendu, on sent que le réalisateur, pourtant souvent "rhyparographe » - ou peintre de choses triviales, obscènes, dans l'Antiquité -, ne peut pas aller aussi loin qu'il le voudrait, à cause de la censure avec laquelle il a été obligé de négocier pied à pied pendant des semaines ${ }^{34}$, ce qui expliquera, plus tard, l'exacerbation et en quantité et en précision de ces corps masculins dans les films suivants, ceux d'après la disparition de la censure, avec des plans sur les sexes des garçons ou sur des coïts gays - mais aussi hétérosexuels, commerce oblige -, depuis El diputado (1978) jusqu'au diptyque de El pico (I et II) (1983 et 1984), en passant par Navajeros (1981) et Colegas (1982). En cela, la scène 
du coït, près du lac, entre Miguel et Carmen nus subsume dans Los placeres ocultos cette volonté de "faire plaisir" à tout le monde, y compris les censeurs, puisque le réalisateur filme la scène en reculant peu à peu de plus en plus, jusqu'à ce que les deux amants nus ne soient plus qu'un point dans l'immensité du paysage.

En conclusion, nous voudrions d'abord rappeler avec Michel de Certeau que :

N'importe quelle historiographie [...] reste toujours le symptôme ou le drapeau du milieu qui l'élabore. [...] [L]e discours historiographique impose comme histoire de la société une Tautologie qui fait que toujours "les mêmes" (ceux qui écrivent) sont les auteurs, les lecteurs et les privilégiés de ces études. Tout le « reste» est silencieusement réprimé par ce cercle du « même " ${ }^{35}$.

Si l'on remplace le verbe " écrire » par le verbe «filmer », on voit que ce que de Certeau reproche à la doxa, c'est l'entre-soi de la réflexion et même du jugement - intellectuel, esthétique, et bien sûr moral -, ce à quoi l'on pourrait ajouter, même, " politique » : un entre-soi auquel s'oppose, par exemple, Eloy de la Iglesia. On insistera alors, justement, sur le soubassement politique, effleuré au début de notre réflexion, de ce film réalisé, après tout, par un communiste, de la Iglesia, à une époque, certes d'ouverture et de changement, mais où l'homosexualité n'était pas - et c'est une litote - l'enjeu premier des luttes de gauche, sinon même un objet de défiance assez partagé par les différents acteurs politiques de cette nation en transition, y compris les plus progressistes.

Le film lui-même n'est pas sans paradoxes et sans subtilités, dans sa diachronie comme dans sa synchronie, pour tout ce qui concerne les enjeux des rapports de classes entre bourgeoisie et prolétariat, entre prostitués du quasi-lumpen, plus ou moins occasionnels, et clients de la bonne bourgeoisie assise, qui sont beaucoup plus réguliers. On a l'impression qu'Eloy de la Iglesia nous lance, en fait, la question suivante : si ces prostitués des cités disparaissaient, du fait d'une redistribution plus égalitaire des fruits de la croissance, ils ne seraient pas obligés de vendre leur corps, ce qui explique, d'ailleurs, dans le film - mais sans l'excuser - la vengeance desdits gigolos de banlieue contre cette bonne et haute société qui l'exploite à tous les niveaux.

Alors, in fine, tout le monde s'assumerait, c'est-à-dire assumerait sa sexualité, par conséquent, il n'y aurait plus d'hétérocentrisme délétère, en Espagne comme ailleurs, aussi ; et si l'on était homosexuel, cette sexualité-là serait une sexualité comme une autre, à partager sans plus de transaction financière, avec un pair, un semblable, pas un banlieusard stipendié car en manque de pesetas.

C'est sans doute pour cette raison que le cinéaste a choisi dans Los placeres ocultos une stratégie de présentation et de visibilité d'un gay viril, en opposition à la prolifération, dans ces années-là d'ouverture et de réforme, de la présence filmique de gays souvent efféminés, comme avant dans le cinéma franquiste ${ }^{36}$. Ce choix de présenter un homosexuel d'aspect viril fait, dès ce film, et fera d'Eloy de la Iglesia plus tard, un réalisateur distinct des autres, parfois attaqué pour cette raison même, alors que son audace, à l'époque, est patente. En effet, par ce choix même, et sous couvert de faire des films mainstream qui n'oublient pas la provocation du destape des corps, il offre aussi au spectateur, quelle que soit son orientation sexuelle, à la fois les corps des femmes et, pour la première fois, ceux des hommes. 
Notre réalisateur crée donc par ce film à la fois une "allégorie politique et un défi artistique $^{37}$ ». Le militantisme d'Eloy de la Iglesia ne réside donc peut-être pas seulement dans cette pédagogie et/ou ce manifeste de l'égalité revendiquée des désirs sexuels, mais dans sa monstration crue à la nation, par le biais de son cinéma « du samedi soir ", de personnages gays à la fois "acceptables ", et qui s'acceptent, déjà, eux. Et ce, quelques années avant un Pedro Almodóvar qui, lui, sera encore différent, avec ses protagonistes labiles et non réductibles à une quelconque taxinomie, dans des films où circuleront, fluides, toutes les formes du désir, sans étiquette, selon une loi qui n'appartient qu'à lui, le désir. Mais c'est une autre histoire... quoique?

\section{BIBLIOGRAPHIE}

Aguilar, Carlos, Devesa, Dolores, Losilla, Carlos et al., 1996, Conocer a Eloy de la Iglesia, SaintSébastien, Filmoteca Vasca/Festival Internacional de Cine de Donostia-San Sebastián.

Angot, Charlotte, 2011, Dimensiones y valor del compromiso en Los placeres ocultos (1976 [sic]) y El diputado (1978), Mémoire de Master (inédit) sous la dir. de N. Noyaret, Caen, Université de Caen.

Berzosa, Alberto, 2011, «El espacio homosexual durante la transición según de la Iglesia: ni a la izquierda ni a la derecha, fuera del armario », dans M. C. Chaput (dir.), Masculin-féminin en transition. Espagne - 1970-1986, Nanterre, Presses universitaires de Paris Nanterre, p. 201-217. Berzosa, Alberto, 2014, Homoherejías filmicas: cine homosexual subversivo en España en los años setenta y ochenta, Madrid, Brumaria.

Blanchot, Maurice, [1955] 1988, L'Espace littéraire, Paris, Gallimard.

Bouchet, Thomas, 2014, Les Fruits défendus. Socialismes et sensualité du XIX siècle à nos jours, Paris, Stock.

Cervulle, Maxime et Rees-Roberts, Nick, 2010, Homo Exoticus. Race, classe et critique queer, Paris, Armand Colin/Bry-sur-Marne, INA.

Chamouleau, Brice, 2011, «L'Affaire Jeremy Thorpe : l'homosexualité en Espagne et le miroir britannique dans la transition à la démocratie », dans M. C. Chaput (dir.), Masculin-féminin en transition. Espagne - 1970-1986, Nanterre, Presses universitaires de Paris Nanterre, p. 185-199. Curopos, Fernando, 2014, « João Miguel Fernandes Jorge : pour un autre art du portrait », Les Langues Néo-Latines, $\mathrm{n}^{\circ}$ 369, p. 123-134.

Feenstra, Pietsie, 2006, Les Nouvelles Figures mythiques du cinéma espagnol (1975-1995). À corps perdus, Paris, L'Harmattan.

Florido Berrocal, Joaquín, Martín-Cabrera, Luis et Matos-Martín, Eduardo (dir.), 2015, Fuera de la ley. Asedios al fenómeno quinqui en la Transición española, Grenade, Comares.

Huard, Geoffroy, 2016, Les Gays sous le franquisme. Discours, subcultures et revendications à Barcelone, 1939-1977, Villeurbanne, Orbis Tertius. 
Le Vagueresse, Emmanuel, 2009, « De quelques manipulations dans El diputado (1978) d'Eloy de la Iglesia : séduction, mensonge et politique sous la Transition ", Les Cahiers du GRIMH, $\mathrm{n}^{\circ}$ 6, p. 357-371.

Le Vagueresse, Emmanuel, 2012, « Travestis, transsexuels et homosexuels dans le cinéma espagnol de la Transition démocratique (1975-1982) : une interrogation à la nation ", Mélanges de la Casa de Velázquez, nº 42-2, p. 125-141, [En ligne], http://journals.openedition.org/mcv/4607, consulté le 28 novembre 2019.

DOI : $10.4000 / \mathrm{mcv} .4607$

Le Vagueresse, Emmanuel, 2014, «Galopa y corta el viento : histoire d'un film impossible (sur un synopsis inédit d'Eloy de la Iglesia) », dans B. Castanon-Akrami, E. Le Vagueresse et C. OrsiniSaillet (dir.), Littérature et Cinéma. Allers-retours : Culture hispanique contemporaine, coll. « Hispanística XX », nº 31, Villeurbanne, Orbis Tertius, p. 163-181.

Le Vagueresse, Emmanuel, 2016, «Violences et humiliations sexuelles dans Juego de amor prohibido (1975) d'Eloy de la Iglesia : entre voyeurisme et politique sous le franquisme finissant », Les Cahiers du GRIMH, $\mathrm{n}^{\circ}$ 9, p. 257-265.

Malaparte, Curzio, [1949] 2013, La Peau [La pelle], Paris, Gallimard.

Melero Salvador, Alejandro, 2010, Placeres ocultos. Gays y lesbianas en el cine español de la transición, Madrid, Notorious/Cáceres, Fundación ReBross.

Melero Salvador, Alejandro, 2017, Violetas de España. Gays y lesbianas en el cine de Franco, Madrid, Notorious.

Mira, Alberto, 1999, Diccionario de cultura homosexual, gay y lésbica, Barcelone, La Tempestad.

Mira, Alberto, 2004, De Sodoma a Chueca. Una historia cultural de la homosexualidad en España en el siglo XX, Barcelone, Egales.

Mira, Alberto, 2008, Miradas insumisas. Gays y lesbianas en el cine, Barcelone, Madrid, Egales.

Montero, Laureano, 2014, Le Cinéma d'Eloy de la Iglesia : marginalité et transgression, Thèse de Doctorat (inédite), Dijon, Université de Bourogne.

Ory, Pascal, 2004, L'Histoire culturelle, Paris, PUF.

Sánchez Cortina, Javier et Cortina de la Calle, Teresa, 2016, « Cine al margen. Reflexión sobre la filmografía quinqui de Eloy de la Iglesia », dans J. González del Pozo (dir.), Márgenes del cine y periferias de la sociedad, Valladolid, Lex Artis, p. 35-83.

Smith, Paul Julian, 1998, La ley del deseo. La homosexualidad en la literatura y el cine español 1960-1990, Barcelone, La Tempestad.

\section{NOTES}

1. «Le titre est un hommage au poète Luis Cernuda, dont le recueil Los placeres prohibidos avait été inspiré par sa passion dévorante pour un jeune gigolo. Ces poèmes [...] étaient un cri de révolte contre une société corrompue et répressive et une célébration du désir et de l'homoérotisme. " À lire dans l'excellente étude de Montero (2014 : 117), thèse définitive à laquelle on se reportera, d'ailleurs (116-126), pour tout ce qui concerne la genèse, les problèmes avec la censure - l'auteur y explique que les atermoiements de deux mois de celle-ci « arrang[èr]ent » finalement le réalisateur, qui attendait que la censure disparaisse totalement pendant ce temps-là, en même temps 
qu'il rendait publics ses problèmes avec elle, afin de servir (la publicité pour) le film et la réception par la presse dudit film, ainsi que la bibliographie générale sur le réalisateur (543-623), dont celle concernant les rapports de censure sur notre film (543) et la réception par la presse (591-592) : sans surprise, les journaux progressistes furent plutôt laudateurs et les conservateurs beaucoup moins, à quelque nuances près. Il existe une importante bibliographie sur le cinéma d'Eloy de la Iglesia, que nous mentionnerons ici ou là sans prétention à l'exhaustivité. Mais nous renvoyons immédiatement, en ce qui concerne ce film-ci, à des contributions intéressantes comme les suivantes: dans Mira, voir d'abord l'entrée "Los placeres ocultos" dans son dictionnaire Para entendernos (1999 : 576-577) ; puis, ses développements sur le film - et d'autres postérieurs du même réalisateur - dans son essai Miradas insumisas (2008: 401-409) ; et enfin, d'autres développements sur ledit film dans son essai De Sodoma a Chueca (2004: 503-507). Citons aussi l'autre grand spécialiste espagnol de la question gay, Melero Salvador, dans son essai passionnant, Placeres ocultos (2010), particulièrement le sous-chapitre «La buena educación: Los placeres ocultos y el activismo pedagógico » (222-235). En France, il y a aussi plusieurs travaux d'envergure, que nous citerons, auxquels nous ajoutons le récent mémoire de Master soutenu à l'université de Caen, en partie consacré à ce film, par Angot (2011).

2. Ce film sera même l'un des derniers à connaître autant de problèmes avec la censure, de toute façon restreinte dès février 1976 par la suppression de la censure "préalable » au tournage des films. Pour ce qui est de l'influence du politique, notamment de la censure, sur la création artistique, nous renvoyons au sous-chapitre «Facteurs politiques » de l'ouvrage d'Ory (2004: 66-73).

3. Sur le lien plus général entre les théories socialistes et la question des plaisirs sensuels, voir l'essai stimulant de Bouchet (2014).

4. Chiffre donné par le ministère de la Culture espagnol, sujet à caution comme tous les chiffres de fréquentation des cinémas à une époque où l'informatisation n'était pas encore de mise pour la collecte des données officielles. En revanche, quelle que soit l'authenticité, cette fois, de la somme rapportée par les recettes, elle est, même minorée, excellente, avec plus de cent millions de pesetas en quatre mois d'exploitation, pour un budget de seulement vingt millions de pesetas injectées par le producteur.

5. Voir sur ce sujet de masculinité, sexualité et nation, l'article de Chamouleau (2011: 188) : «[...] [L'affaire en question, où sont mêlées politique et homosexualité] [...] tout en problématisant et en déplaçant la frontière mouvante entre les catégories du privé et du public, se déploie progressivement pour constituer une véritable "question sexuelle", entendue au sens d'Éric Fassin: "[elle aborde] dans un langage sexuel d'autres questions politiques" [Éric Fassin, Le Sexe politique. Genre et sexualité au miroir transatlantique, 2009] [...]. L'Affaire Thorpe serait alors paradigmatique: elle s'inscrit dans un réseau significatif qui dépasse largement le cadre strict de la sexualité et du genre, et installe l'événement dans un réordonnement politique autour du fonctionnement démocratique du Royaume-Uni » à lire dans l'ouvrage coordonné par Chaput (2011), ou aussi l'article du même volume sur Eloy de la Iglesia écrit par Berzosa (201-217), et, du même chercheur, une remarquable étude sur le cinéaste (2014: 184-206). On peut se reporter également à notre propre article (Le Vagueresse 2012), qui brosse un panorama de la représentation de l'homosexualité - masculine - dans le 
cinéma de la Transition, à lire dans l'excellent ouvrage coordonné par Aguado et Yusta (2012).

6. Une modification, signée par le chef du gouvernement Adolfo Suárez, de la « Loi sur la Dangerosité et la Réhabilitation Sociale » franquiste écarta les homosexuels de ce texte répressif uniquement le 26 décembre 1978, pour une entrée en vigueur le 11 janvier 1979, les derniers homosexuels emprisonnés étant libérés cette année-là, soit, tout de même, quatre ans après la mort du dictateur.

7. On se reportera sur ce sujet à Feenstra (2006:192) : «[En Espagne, à partir de la Transition] le cinéma espagnol témoigne des nouvelles images du corps homosexuel : le corps féminisé permettait la visibilité, bien lié à la marginalité de l'homosexualité. L'opposition binaire, mise en place par le contraste entre le stéréotype et l'image exemplaire (prototype), interrogeait deux valeurs archétypales, c'est-à-dire la famille et la virilité masculine. [...] [G]râce à la modification des lois pendant la période de la Transition [donc, pour Los placeres ocultos, un peu plus tard, ce qui fait de ce film un précurseur], le cinéma national reflète le rejet du stéréotype [de la folle] en introduisant une image masculine de l'homosexuel, homme exemplaire de bonne volonté et intelligent. »

8. Nous avons déjà étudié le cinéma d'Eloy de la Iglesia, à plusieurs époques de sa production. Nous renvoyons donc à nos articles pour éviter de répéter l'analyse de certains éléments (Le Vagueresse 2009, 2014, 2016) ; Sur la mode du cinéma " quinqui » ou «de voyous » - où les protagonistes sont des jeunes voyous des cités - pendant la Transition, voir par exemple Florido Berrocal, Martín-Cabrera et Matos-Martín (2015) et, pour ce qui concerne Eloy de la Iglesia, voir l'article de Sánchez Cortina et Cortina de la Calle (2016).

9. Voir Melero Salvador (2017: 300) : «Pour [Linda] Williams, l'ellipse qui sépare le début de la scène de sexe [...] de son résultat [...] fonctionne comme des "points de suspension". Pour Williams, "les ellipses se font sentir davantage lorsque ce qu'elles éludent est un acte sexuel". » Nous traduisons toutes les citations extraites d'ouvrages écrits en espagnol.

10. Voir le studio français de porno gay Citébeur et sa représentation des fantasmes du consommateur de ce genre de productions dans ces films concernant les jeunes gens de banlieue et/ou de cité, "[e]n situant socioéconomiquement les "lascars" et "racailles" (par le biais du style, de nombreuses références au travail ou au non-travail, du recours à des décors urbains)» (Cervulle et Rees-Roberts 2010:77), ainsi que la totalité du chapitre "Kiffe la racaille », sur les stratégies des studios de porno gay vis-à-vis de ces fantasmes sur les garçons des « técis » (71-79).

11. Tapissée d'images de femmes nues, autre contraste avec l'intérieur d'Eduardo, où l'on aperçoit des tableaux de maîtres et un tableau d'homme nu, mais artistement dessiné.

12. On se situe donc In Gay Madrid, d'après le titre du film américain de 1930 signé Robert Z. Leonard avec Ramón Novarro, même s'il s'agit pour nous ici d'un clin d'œil, ce film ne parlant pas du milieu gay de l'époque, mais du Madrid de tous les plaisirs et de toutes les fêtes (et alors que le Mexicain R. Novarro était pourtant un acteur homosexuel dans le placard, comme le personnage d'Eduardo dans Los placeres ocultos).

13. L'ouvrage de référence sur le cinéaste qu'est Conocer a Eloy de la Iglesia publie sa réponse à une question sur le côté « pamphlétaire » du film : «À ce moment-là c'était 
inévitable. D'abord, parce que c'était le premier film où je pouvais parler ouvertement de ce sujet. Et, ensuite, parce qu'il me semblait qu'il fallait le traiter jusque dans ses dernières conséquences, avec la nécessité de créer un tramage, disons, théorique. Et d'autre part, commençait à surgir un certain affrontement entre mon militantisme politique et la manière dont ce militantisme affectait ma vie personnelle. [...] Je me sentais dans l'obligation intime d'affronter ces problèmes " (Aguilar, Devesa, Losilla et al. 1996: 132-133). Nous soulignons pour le rapport à l'histoire personnelle du réalisateur, qui était encarté au Parti communiste espagnol, et à la tension, à l'époque, chez lui, entre son engagement et sa vie intime, entre politique et Eros, précisément, alors que les communistes « orthodoxes » n'avaient pas encore, loin s'en faut, procédé à leur aggiornamento quant à la vision de " péché capitaliste » accolé à l'homosexualité.

14. Voir Malaparte ([1949] $2013: 122)$ et tout le chapitre intitulé «Les roses de chair» (117-173).

15. Sur les prémices de ce combat collectif gay, encore balbutiant en 1977, on renvoie, par exemple, au chapitre «Socialización (I): el movimiento de liberación gay hasta 1983 ", dans Mira (2004 : 473-494).

16. Ce genre de cité de la périphérie madrilène, autrefois rurale, qui sera l'apanage des films de «quinquis » durant ces années de la Transition, dont font partie les longs métrages d'Eloy de la Iglesia, apparaît déjà dans l'un des premiers succès du cinéaste, $L a$ semana del asesino (1972), avec un personnage d'homosexuel, pas encore totalement explicite, censure oblige. Dans ce film, on voyait déjà un personnage vivre, non pas dans la cité nouvelle, mais dans une humble maison, rattrapée, atteinte, mangée bientôt par ces nouvelles constructions gigantesques de béton pour la plèbe. De la Iglesia suit ainsi, à sa manière, le « fûkeiron » ou « théorie du paysage » élaborée à la fin des années 1960 par des cinéastes japonais, dont Masao Adachi, qui stipulait que tous les paysages que l'on rencontre au quotidien reflètent les structures du pouvoir en place et que la caméra doit scruter ces paysages pour y déceler lesdites structures oppressives, notamment dans les périphéries urbanisées.

17. C'est-à-dire un hétérosexuel qui couche avec des hommes moyennant finance.

18. Rappelons que chez les anciens Grecs, un éromène est un adolescent engagé dans une relation pédérastique avec un homme adulte, appelé éraste. Ce statut social reconnu, où l'éromène recevait une éducation physique et intellectuelle, était extrêmement codifié et réservé, de facto, à une certaine élite aristocratique.

19. Car Raúl a connu le même chemin que Miguel, en étant, de plus, homosexuel, ce qu'étudie bien Smith dans son classique et pionnier Las leyes del deseo (Smith 1998 : 144) : «Los placeres ocultos n'offre aucun exemple de relation réciproque entre hommes du même statut. Même Raúl [...], tout aussi cultivé et bourgeois que son ex-amant, a été un jour comme Miguel, un jeune homme pauvre illicitement introduit dans le travail par Eduardo", et aussi : "Raúl attaque Eduardo: il y a eu une époque où [Eduardo] achetait simplement les corps des garçons : maintenant, il achète leur vie. Ce dernier réplique qu'il est en train d'essayer de former une "espèce de famille" avec le jeune couple et qu'ainsi il évitera la solitude dans ses vieux jours. Raúl, le révolutionnaire sexuel, répond que lui et ses camarades ne seront pas seuls non plus au moment de la lutte; il est inutile de s'appuyer sur les efforts individuels. » On lira avec profit, de Smith, tout le chapitre intitulé «El Cine de transición de Eloy de la Iglesia » (133-169), dont le sous-chapitre «Los placeres ocultos: el triángulo erótico » (140-147). 
20. Mais le fait que, quelques minutes après, dans une autre scène, Eduardo pose sa main sur la jambe de Miguel, sans concupiscence cette fois, et que ce dernier ne la retire pas, montre le progrès du jeune homme vis-à-vis de ce qui était auparavant chez lui une véritable " panique homosexuelle».

21. Celle qu'Eduardo lui avait offerte, vers le début de leur relation, quand celui-ci voulait encore « acheter ", consciemment ou pas, le jeune homme pauvre par ce cadeau de prix.

22. Et qui rappelle le « ¿Quita, marica!» du coming out d'Eduardo auprès de Miguel, comme s'il y avait régression a priori dans l'acceptation de l'homosexualité par Miguel.

23. Même s'il fait un geste en demandant aux deux vigiles de la banque de ne pas tenter de maitriser le jeune homme par la force.

24. En effet, vu sa position élevée dans la hiérarchie sociale, un gay comme Eduardo ne risquait pas grand-chose, à la différence du "petit peuple». Voir par exemple la remarquable étude de Huard (2016), notamment le sous-chapitre «Homosexualité et délinquance: la justice franquiste de classe » (54-63), et encore en particulier «L'augmentation de l'“homosexualisme”, 1970-1977 » (57-63).

25. La fin est aussi ouverte que celle de El diputado, qui s'achève au moment où le protagoniste, un député gay va faire son coming out devant ses collègues de l'Assemblée nationale espagnole, les « Cortes ».

26. On pense évidemment au grand succès commercial que fut No desearás al vecino del quinto (1972), de Tito Fernández, et au personnage d'Antón (Alfredo Landa), un couturier qui, dans le film, joue les folles pour mieux séduire les femmes sans éveiller les soupçons de leur mari (sic).

27. Rappelons que le choix de l'acteur Simón Andreu - comme plus tard de José Sacristán dans El diputado - pour ce rôle d'homosexuel viril obéit à cette même stratégie, de la part du réalisateur, ici en faisant jouer un gay par un comédien notoirement hétérosexuel, pour que l'identification soit possible au maximum avec le spectateur mâle non gay.

28. Et, même si c'est un hasard, du prénom du Généralissime lui-même.

29. Cette prise de conscience commence pour lui à se faire, aussi, à partir de la mort de sa mère, dans la diégèse, selon un cliché freudien bien connu, que ne discute pas Eloy de la Iglesia.

30. Comme dans tous les films d'Eloy de la Iglesia, ce qui déplaisait fortement à la critique conservatrice en ce temps de destape, mais faisait venir les spectateurs, ce qui était, aussi, un souhait assumé du réalisateur. Le destape espagnol n'est, bien entendu, pas la seule référence à mobiliser ici, mais, de manière plus générale, le développement du cinéma érotique dans le monde occidental, dont le film reprend plusieurs clichés (pubis et poitrines de femmes montrés frontalement, choix d'une musique «porno soft ", etc.).

31. Les personnages féminins mériteraient, bien évidemment, une étude spécifique. On se contentera de faire remarquer, ici, qu'ils sont tous hétérosexuels et s'inscrivent dans des rapports relativement stéréotypés, eux aussi, quant à leur caractérisation et leur traitement : cf. la fiancée compréhensive, la mère aimante, la maîtresse manipulatrice et adultère, en tant que contrepoint/complément de la masculinité. 
32. Ce fut d'ailleurs la même stratégie pour El diputado, l'affiche montrant le visage du député lunetté, avec dans le reflet des lunettes une scène d'amour à trois - un jeune homme, le député et son épouse Carmen - propre à rassembler les désirs autant hétérosexuels qu'homosexuels, dans un même goût, aussi, de racolage commercial, qui misait peut-être sur le soft power, ou changement progressif des mentalités par une sorte d'entrisme des esprits du « spectateur moyen », dont on a déjà parlé plus haut.

33. On ne développera pas les plans énamourés du réalisateur sur les jeunes prostitués torse nu de cette cité, gimmick de tous les films postérieurs d'Eloy de la Iglesia, et déjà visibles ici.

34. Les négociations avec la censure sont détaillées, à partir des rapports de censure eux-mêmes, par Montero (2014 : 119-123), en de passionnants développements.

35. Selon Michel de Certeau (La Culture au pluriel, 1974), cité par Curopos (2014: 12). C'est l'auteur qui souligne.

36. Mais dans le but de faire accepter ces gays bien différents des "folles" qui provoquaient les rires dans les films de la dictature. Pour autant, le gay efféminé, voire le travesti, rassure les hommes, les Espagnols, la nation espagnole dans son entier, car il est immédiatement reconnaissable, identifiable, assignable et classable. De plus, même s'il existe énormément de personnages homosexuels dans le cinéma de la transition démocratique espagnole, il n'y a pas dans ce cinéma uniquement des personnages positifs ou des contenus qui les défendent, ni clairement, ni même, d'ailleurs, en demi-teinte ou hypocritement. On y voit, en fait, tout l'éventail des visions de l'époque sur l'homosexualité : on pense au réalisateur Ignacio M. Iquino et à sa croisade homophobe réactionnaire, pour citer un cinéaste opposé au progressisme sexuel (voir, entre autres, son Fraude matrimonial, 1976).

37. "Alegoría política y desafío artístico ", d'après le titre - que nous détournons éhontément - de l'exposition au Museo del Prado, Madrid, du 21 janvier au 4 mai 2014.

\section{RÉSUMÉS}

Le cinéaste espagnol Eloy de la Iglesia a toujours été engagé contre la doxa et les dogmes, quels qu'ils soient, moraux, sensuels, sexuels, mais aussi politiques, nationaux, patriotiques. Avec ce dixième long-métrage de fiction, Los placeres ocultos, le réalisateur brave plus ouvertement que jamais la censure (post-)franquiste moribonde, en proposant l'histoire d'Eduardo, bourgeois homosexuel dans le placard mais entouré de prostitués, qui tente soudain de prendre sous son aile un jeune des cités, Miguel, pour le faire progresser dans la société, sublimant - parfois avec peine - son désir pour l'hétérosexuel, afin de le faire accéder aux sphères supérieures de la réussite sociale.

Retenu plusieurs mois par la censure, et sorti enfin sur les écrans en mai 1977, ce film fait date dans la représentation cinématographique commerciale de la "question gay ", à un moment où tout semble devenir possible. Questionnant via un cinéma faussement mainstream l'hétérocentrisme de la nation espagnole au tout début de la transition démocratique, ce film est reçu non sans réticences par la presse spécialisée, mais avec un grand succès d'audience. Il 
annonce la profusion de films plus ou moins militants, mais aussi voyeurs, sur le sujet à cette époque, faisant de l'acceptation de l'homosexuel «moyen»- et non plus de la «folle »- le symptôme de la nouvelle liberté de tout un pays en voie vers la démocratie.

The Spanish film director Eloy de la Iglesia has always been an opponent of Doxa and dogma of any kind, be they moral, sensual, sexual, but also political, national or patriotic. In Los placeres ocultos, his tenth feature-length film, the director defies more openly than ever the moribund (post-)Francoist censors, through the story of Eduardo, a closet homosexual but who is surrounded by prostitutes, and who suddenly tries to take under his wing a youth from a housing estate, Miguel, in order to help him climb the social ladder, while rising above his desire for the heterosexual-sometimes with difficulty-so as to help him attain the higher spheres of social success.

Held back by the censors for several months, until its eventual release in May 1977, this film is a landmark for the cinematographic depiction in commercial films of the "gay question," at a time when all seemed possible. Questioning the heterocentrism of the Spanish nation via a seemingly mainstream film at the onset of the Demographic Transition, the film received a cool reception from the critics, but was a box office hit. It ushered in a profusion of films on the subject that were more or less militant, but also voyeuristic, making the acceptance of the "ordinary" homosexual-rather than the "queer"-the symptom of the newfound liberty of an entire country moving towards Democracy.

\section{INDEX}

Keywords : cinema, Spain, Democratic Transition, censorship, homosexuality

Mots-clés : cinéma, Espagne, transition démocratique espagnole, censure, homosexualité

\section{AUTEUR}

\section{EMMANUEL LE VAGUERESSE}

Université de Reims Champagne-Ardenne, CIRLEP 